\title{
The first light of the Solar Activity MOF Monitor Telescope (SAMM)
}

\author{
Roberto Speziali ${ }^{1, *}$, Andrea Di Paola ${ }^{1}$, Mauro Centrone ${ }^{1}$, Maurizio Oliviero ${ }^{2}$, Domenico Bonaccini Calia ${ }^{3}$, \\ Luciano Dal Sasso ${ }^{4}$, Marco Faccini, Vincenzo Mauriello ${ }^{4}$, and Luciano Terranegra ${ }^{2}$
}

${ }^{1}$ INAF - Rome Astronomical Observatory, Via Frascati, 33, 00078 Monte Porzio Catone, RM, Italy

2 INAF - Capodimonte Astronomical Observatory, Salita Moiariello, 16, 80131 Naples, Italy

${ }^{3}$ European Southern Observatory, Karl-Schwarzschild-Str. 2, 85748 Garching bei München, Germany

${ }^{4}$ Avalon Instruments, Via dei Rutuli, 101, 04011 Aprilia, LT, Italy

Received 18 June 2020 / Accepted 24 December 2020

\begin{abstract}
SAMM (Solar Activity MOF Monitor) is a ground based robotic instrument that has been developed to study and constantly monitor the magnetic activity of the Sun, focusing on Active Regions (ARs). These regions are characterized by complex magnetic structures that may result in explosive events usually associated with large amount of particle and matter ejections in the space environment. When interacting with the Earth magnetosphere they can represent a threat for our infrastructures both in space (satellites, navigation systems) and on the ground (power plants and electrical grids). Based on Sodium (Na) and Potassium (K) magneto optical filters (MOFs), SAMM provides a "tomographic" view of the magnetic structures delivering high cadence magnetograms and dopplergrams at different heights of the solar atmosphere thus providing a unique dataset with the aim to push forward the current space weather forecasting capabilities. Being able to forecast these events enough in advance (even few hours) is a fundamental task to put in place mitigation strategies to reduce the potential catastrophic impact on vital infrastructures on earth. In this scenario the SAMM observatory has been realized to be a "node" that can be replicated in a world-wide network with the aim to give a continuous coverage of the Sun situation. This project has been initially funded by the Italian Ministry of Economic Development (MiSE) in 2015 through a soft loan grant and its development and operation is carried on within a scientific collaboration between the INAF - Rome and Naples Astronomical Observatories and the Italian small enterprise (SME) Avalon Instruments. After three years of development, SAMM is in the commissioning phase. In this paper we are presenting a final instrument description along with the first light images.
\end{abstract}

Keywords: space weather / ground based instrumentation / magneto optical filters / MOF

\section{Introduction}

Space weather events come in different forms and strengths and, just like weather on Earth, some of these could be highly disruptive. Even if the space weather phenomena intensity is governed by the 11-year solar cycle so that it is possible to predict, at some level, the period when their effects are likely to be most severe, this is not enough. As mankind becomes more reliant on modern technologies (and as infrastructures become more interconnected) a major space weather event (such as flares or coronal mass ejections) could disrupt both civil and military strategic infrastructures such as power grids, aviation and satellite systems. At the same time, the radiation flux peaks connected to space weather represent not only a risk to the operation of modern aircrafts but also to the health of aircrews, passengers and astronauts participating in manned space missions. In this

\footnotetext{
*Corresponding author: roberto. speziali@inaf.it
}

scenario having accurate and timely space weather predictions is the key to deploy mitigation strategies.

Various damages (outages of power plants, grids and the impacts on navigation systems in commercial aviation) due to space weather starting in 1859, the year in which the most powerful event was recorded (Carrington Event), are described in various reports (Lloyd's, 2010, 2013; Kappenman, 2010; SCOR Papers, 2014. Furthermore, since the late 1990s, partial or total losses of satellites have also been recorded (Intelsat, Anik, Galaxy, Sky Terra 1, Spaceway 3, etc.).

Given the awareness of the catastrophic effects that a Carrington-like event would have on the current society (blackouts of weeks are estimated and damages ranging from 0.6 to 2.6 trillion dollars in the case of an impact over USA and Canada), much has been done to improve the observational coverage of solar phenomena both from space and from earth.

Various government agencies have equipped themselves with monitoring and control centers for solar activity (NOAA, 

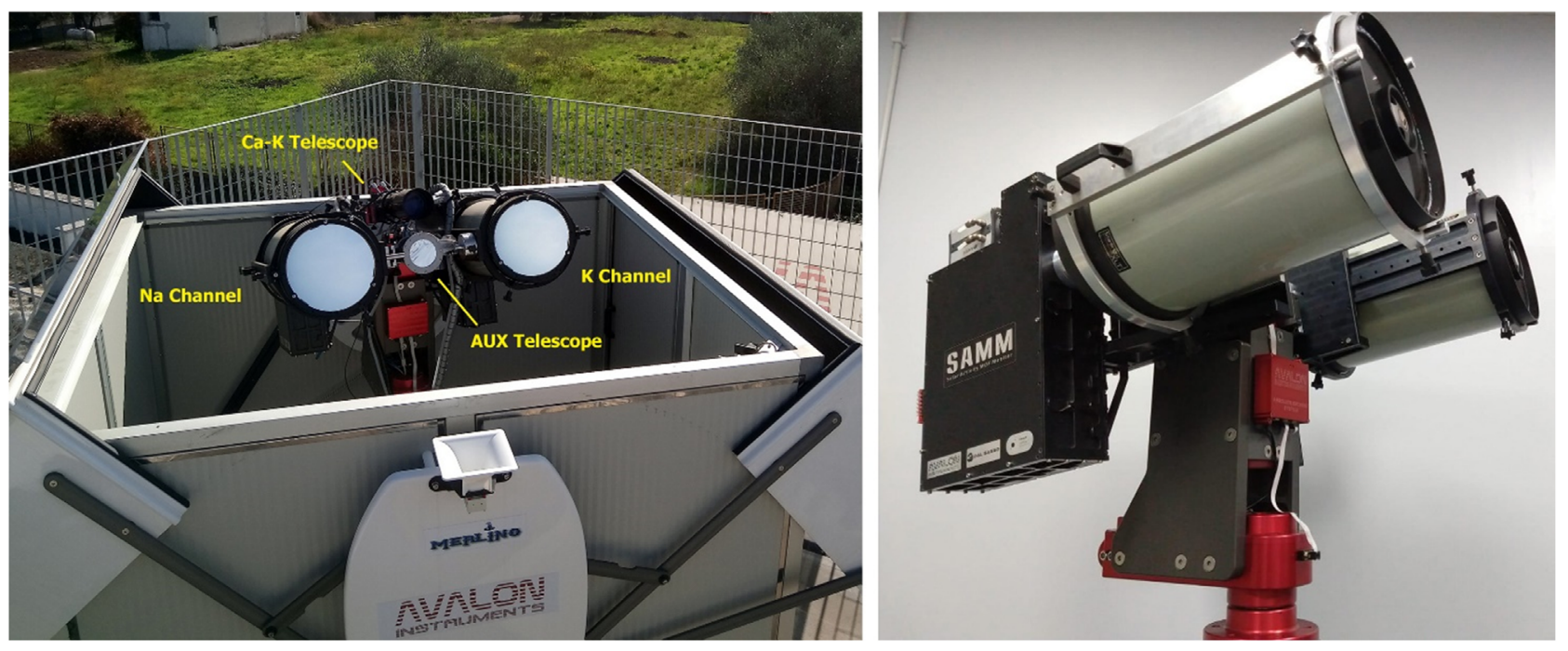

Fig. 1. Left: the first SAMM node installed on top of the Avalon Instrument Headquarters near Rome. On top of the SAMM we have two telescopes: one is equipped with a $\mathrm{Ca}-\mathrm{K}$ filter to preliminary detect and check the pointing on the magnetic structures we want to observe, a smoller one (Aux) is used for the mount setup and can be used also at night since has a removable neutral density filter. Right: the SAMM prototype in the lab.

ESA, NRC, SANSA, SWS) and several nations have implemented structures capable of issuing daily space weather bulletins.

The development of the SAMM fits into this rapidly developing scientific and technological context. SAMM is able to give a direct measurement of the magnetic field simultaneously at two levels and with a high temporal cadence (of the order of $2 \mathrm{~min}$ ) giving an unprecedented set of data to study the magnetic active regions of the Sun.

Recently the SAMM telescope has been added to the INAF Space Weather Instrumentation Network, a strategic Italian infrastructure aimed at space weather monitoring to be used as a basis for a timely information dissemination service useful for the study and mitigation of the adverse impact of severe space weather events on both civil and military infrastructures.

\section{Description of the SAMM}

The SAMM node is based on a binocular instrument in which each channel consists of a $23 \mathrm{~cm}$ diameter telescope. Each telescope is equipped with very narrow-band polarimetric instruments tuned at a specific wavelength (Fig. 1).

The "core" of these instruments is represented by magnetooptical filters (Agnelli et al., 1975; Cacciani \& Fofi, 1978; Cacciani \& Moretti, 1994; Cacciani et al., 1997). They are super narrow band filters $(50 \mathrm{~m} \AA)$ and represent a unique technology of its kind. Unlike instruments based on Fabry-Pérot interference filters or Michelson interferometers, MOFs have an intrinsic high spectral stability due to the fact that their working wavelength is determined by the atomic transition of the inner gas. They can be tuned by changing the temperature that can be easily stabilized within $0.1{ }^{\circ} \mathrm{C}$ hence giving a good sensitivity for magnetic and Doppler measurements (see Table 1). And moreover MOFs compactness, low weight and, above all, the
Table 1. Main characteristics of the SAMM telescope.

\begin{tabular}{ll}
\hline Telescope & Celestron 9.25" EDGE \\
Detector & Zyla 5.5 sCMOS \\
Mount & Avalon Instruments M3 \\
FOV & $8.3 \times 9.8 \mathrm{arcmin}$ \\
Resolution & $0.23 \mathrm{arcsec} / \mathrm{pix}$ \\
Wavelength & $589 \mathrm{~nm}(\mathrm{Na}) \& 770 \mathrm{~nm}(\mathrm{~K})$ \\
Exposure time $\times$ single image & $1-3 \mathrm{~ms}$ \\
Acquisition time $\times$ & $0.8 \mathrm{~s}$ \\
1 set $(=1$ image $\times 4$ LCVR states) & \\
Acquisition time $\times 100$ sets $($ cadence) & $80 \mathrm{~s}$ \\
Sensitivity limit & $\sim 10 \mathrm{Gauss} *$ \\
Resolution doppler limit & $\sim 10 \mathrm{~m} / \mathrm{s} * *$ \\
\end{tabular}

* Expected from Figure 3 of Vogt et al. (1999).

** Expected from Table 1 in Oliviero et al. (2002).

absence of moving parts, make them highly efficient and economical components.

The operating principle the SAMM is based on has already been outlined in the SAMM introductive paper (Stangalini et al., 2018) and is well known since has been used in previous instrumentation like MOTH (Cacciani et al., 2003) and VAMOS (Oliviero et al., 1998; Magrì et al., 2008). This principle can be summarized as follows: each SAMM channel provides magnetograms and dopplergrams by a proper combination of four images taken onto the blue and red side of the two Zeeman circular polarized components $\sigma^{+}$and $\sigma^{-}$of the solar absorption line splitted by the magnetic field. Each component is first selected by a liquid crystal variable retarder (LCVR), then the background is cancelled out by the first MOF cell placed in between two crossed linear polarizers and finally, by modulating a second LCVR in combination with the second MOF called "Wing Selector", the blue and red signals are separated. The difference between these two is proportional to the Doppler 


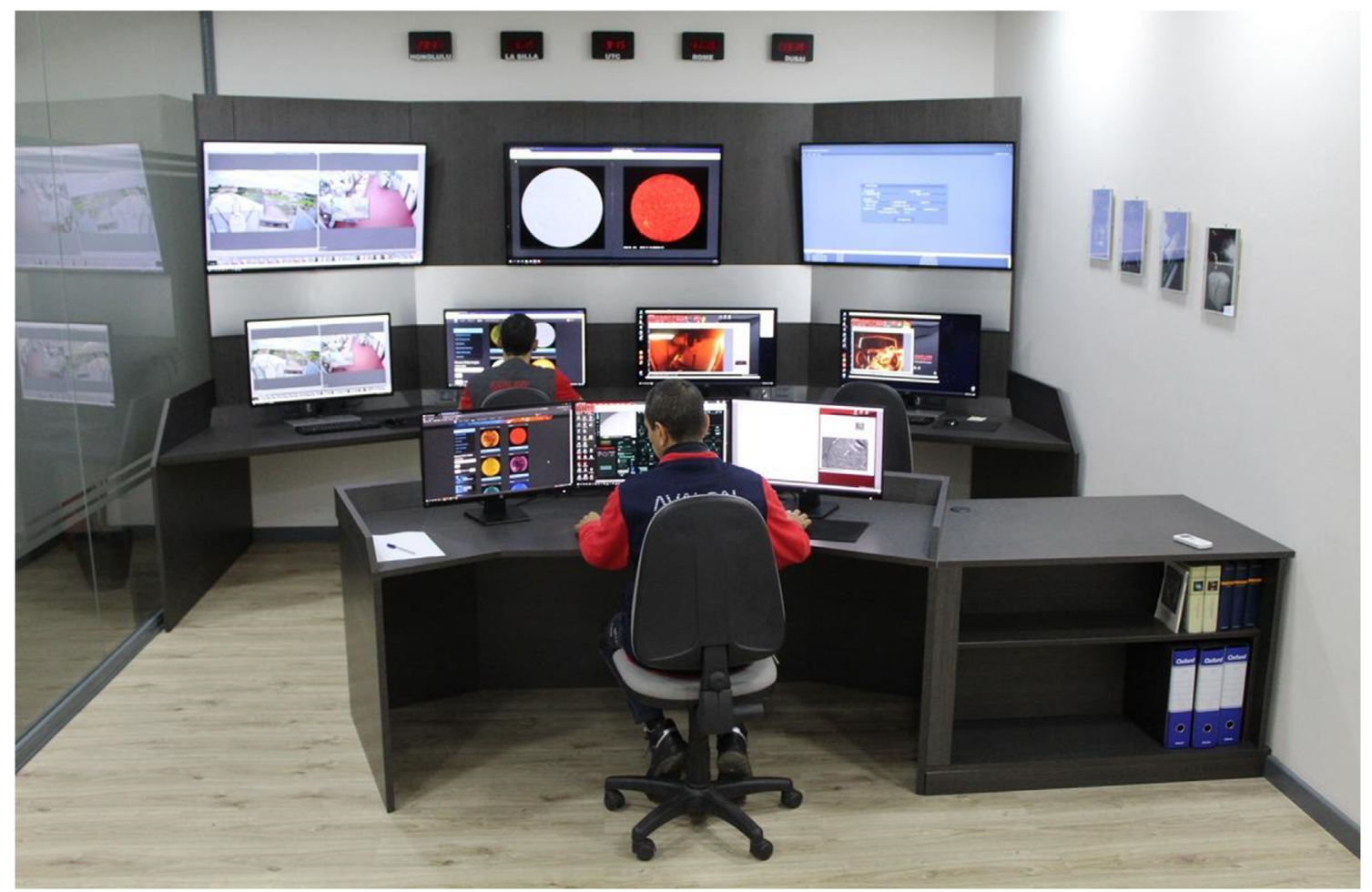

Fig. 2. The Avalon Instrument telescopes remote network control room.

shift of the line, while the separation between the shifts of $\sigma^{+}$and $\sigma^{-}$is proportional to the line of sight (LoS) magnetic field.

With the two acquisition channels based on MOF filters, suitably made to work on the wavelengths NaD2@5889.95 and KD1@7698.96 $\AA$, the SAMM is able to directly and simultaneously measure the LoS magnetic field $(B)$, the intensity $(I)$ and the velocity $(V)$ of the solar plasma at two altitudes: around $200 \mathrm{~km}$ (photosphere) and $700 \mathrm{~km}$ (chromosphere).

Instruments that are currently able to perform magnetograms, such as SDO-HMI (Schou et al., 2012) and GONG (Leibacher et al., 1996), measure the magnetic field exclusively in the photosphere. None of them is currently able to provide simultaneous information at different heights. The use of at least two channels for the simultaneous acquisition of images in different bands, the high temporal cadence ( $80 \mathrm{~s}$ ) and the high spatial resolution $(0.23$ arcsec/pixels), make the SAMM a unique instrument. See table below with the main characteristics of the SAMM summarized.

In addition to the measurement mode and the used technology, the SAMM also emerges in the current instrumental panorama when costs are considered. Thanks to the use of MOFs and relatively low cost optical components, an entire SAMM observing station adds two more orders of magnitude in cost savings if compared to satellites cost and other planned networks (Hill et al., 2019).

The SAMM observational station has been built with the aim to empower a worldwide network of instruments. The SAMM station, in fact, is already configured as a modular product, able to be replicated and displaced to different longitudes to guarantee the surveillance of solar activity continuously over a 24-h period.
In an ideal long-term scenario, a network consisting of a dozen SAMM stations would have a relevant scientific interest enabling INAF, Avalon Instruments and, possibly, any further partner/customer, to produce an exclusive set of data concerning the space weather (Fig. 2).

\section{Upgrades and MOF characterization}

In the past two years the two channels have undergone some major mechanical and optical reconfiguration to solve some critical issues of the original design and to increase the operational and optical efficiency of the system. The reviewed version includes one rotary and translation stage to perform remote tuning of both the entrance linear polarizer position and the focus. The two mylar neutral density filters with a transmission of the order of $10^{-4}$, mounted on the entrance of the two telescopes, have been replaced with two new custom glass neutral filters with a transmission two order of magnitude higher than the mylar ones thus increasing the signal and still reducing the thermal load on the system $(<0.1 \mathrm{~W})$.

A great care has been devoted to the characterization of the MOF filters in both channels. This procedure is fundamental to find the operative temperature of the two filters (the MOF and Wing Selector) to achieve the best compromise between a high throughput and the minimum cross talk between the $\sigma^{+}$and $\sigma^{-}$ wings of the absorption lines.

Our potassium MOF cells have been tested at the Osservatorio Astronomico of Capodimonte on the same optical test bench used for the VAMOS experiment (Fig. 3) while the sodium cells have been characterized at the ESO Technology Division Laser Systems Department (ESO headquarters in 


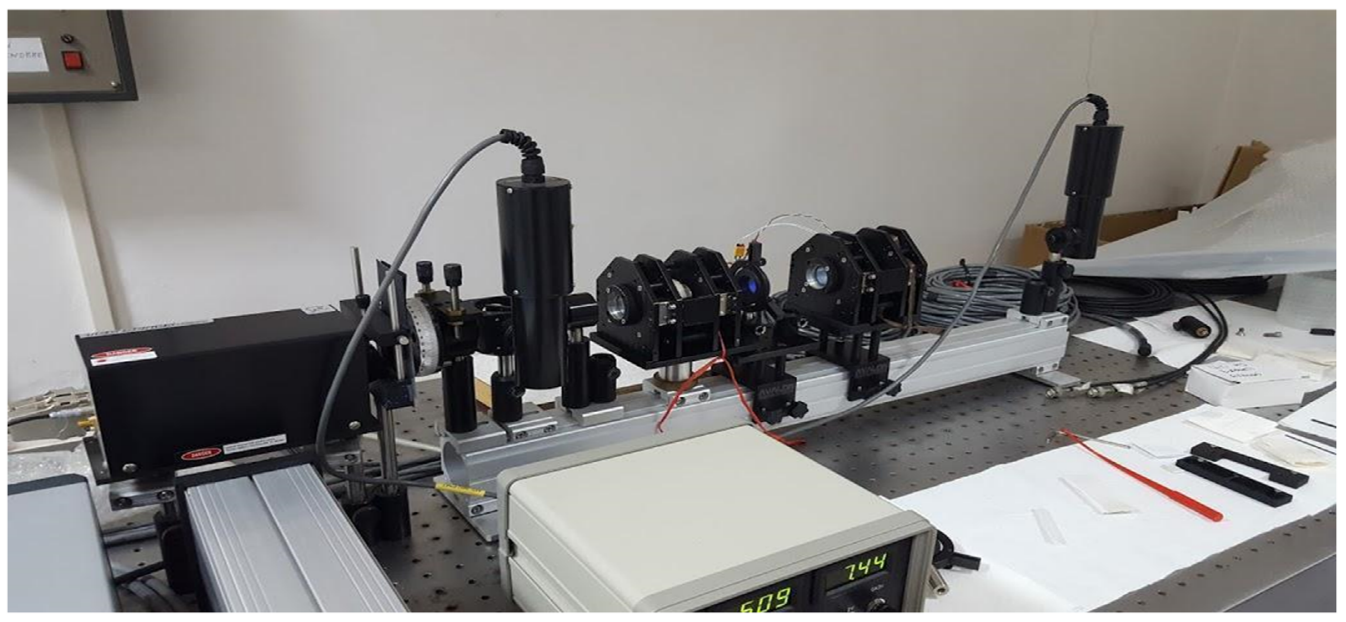

Fig. 3. The MOF-K cells mounted on the laser test facility at INAF-OAC.

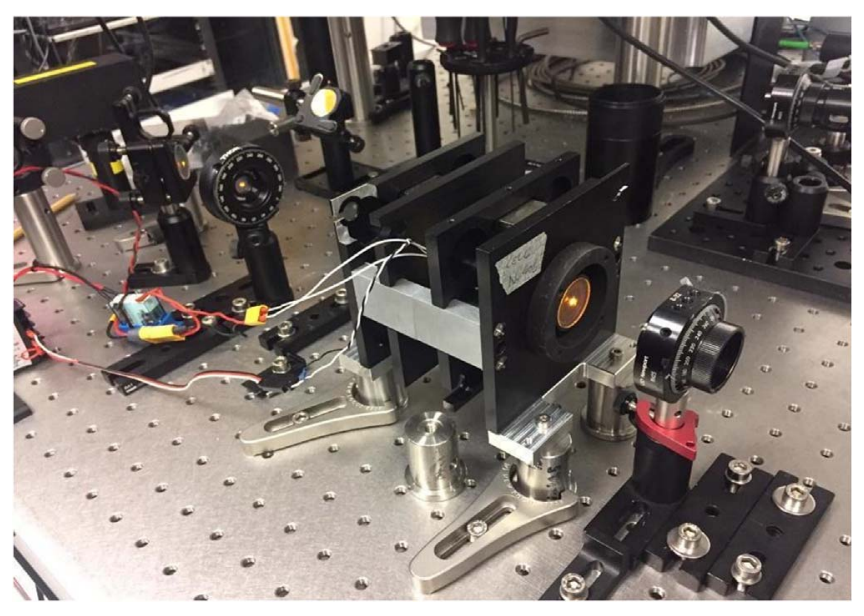

Fig. 4. One of the Na-MOF cell mounted on the optical bench at the ESO-LGS Lab.

Garching) where we are developing a new kind of MOF based instrumentation to be applied to Laser Guide System (LGS) for both astronomical and satellite communication applications (Fig. 4).

In both cases, we have performed several wavelength scans by varying the operative temperature and magnetic fields of the filters to find the best combination of both MOF and Wing Selector.

These best operative temperature depends on many factors and vary from cell to cell depending on reservoir geometry, magnetic field configuration, density of material and the pressure of the buffer gas. For in use cells, the best compromise to optimize the transmission efficiency in the two polarization states are listed in the Table 2.

The results of the transmission profiles versus the working temperature of the MOF and WS are displayed in the figures below respectively for the $\mathrm{Na}$ (Fig. 5) and K (Fig. 6) channels.

The central maximum in the middle of the Figure 6-left is present only below about $120^{\circ} \mathrm{C}$, and is due to the convolution of the Macaluso-Corbino effect on both the Zeeman components in correspondence of the central wavelength. Increasing
Table 2. Operative temperatures of the MOFs used in the SAMM telescope.

\begin{tabular}{lccccc}
\hline \multirow{2}{*}{ Cell type } & \multicolumn{2}{c}{ MOF } & & \multicolumn{2}{c}{ Wing selector } \\
\cline { 2 - 3 } \cline { 6 - 7 } & Temp & Mag. field & & Temp & Mag. field \\
\hline $\mathrm{Na}$ & $250^{\circ} \mathrm{C}$ & $2000 \mathrm{G}$ & & $250{ }^{\circ} \mathrm{C}$ & $3700 \mathrm{G}$ \\
$\mathrm{K}$ & $115^{\circ} \mathrm{C}$ & $1240 \mathrm{G}$ & & $150{ }^{\circ} \mathrm{C}$ & $2480 \mathrm{G}$ \\
\hline
\end{tabular}

the temperature, the Zeeman absorption dominates the Macaluso-Corbino effect and the peak disappears, in agreement with theoretical and experimental MOF transmission profiles (Severino et al., 2007). The central peak is dependent also from the cell magnetic field. In fact at higher magnetic field the Zeeman splitting is bigger and, at fixed temperature, the transmission peak due to the overlap of the Macaluso-Corbino effect dominates on the Zeeman absorption at the central wavelength. For this reason, to avoid the central peak detected in the MOF-k low temperature profile (Fig. 6 left), we had to lower the magnetic field down to $1240 \mathrm{G}$. The final parameters of our MOFs are indicated in Table 2 and correspond to the red curves in Figures 5 and 6. With this setup we can observe the solar photosphere at about $200 \mathrm{~km}$ (Tomczyk et al., 1995) and the chromosphere at about $700 \mathrm{~km}$.

\section{Control software}

There are three software packages to control the whole SAMM station: one for the Merlino dome, one for the StarGo mount and another to operate the two MOF polarimeters.

The first two are proprietary softwares developed by Avalon Instruments and are widely used in their products. The GUIs have been slightly customized for the SAMM operations. The Merlino is a full sky enclosure equipped with a custom electronics to manage multiple power supply lines and the housekeeping motors. The mount is an alt-az prototype equipped with high resolution absolute encoders that uses the same belt drive technology of the smaller ones for the amateur 

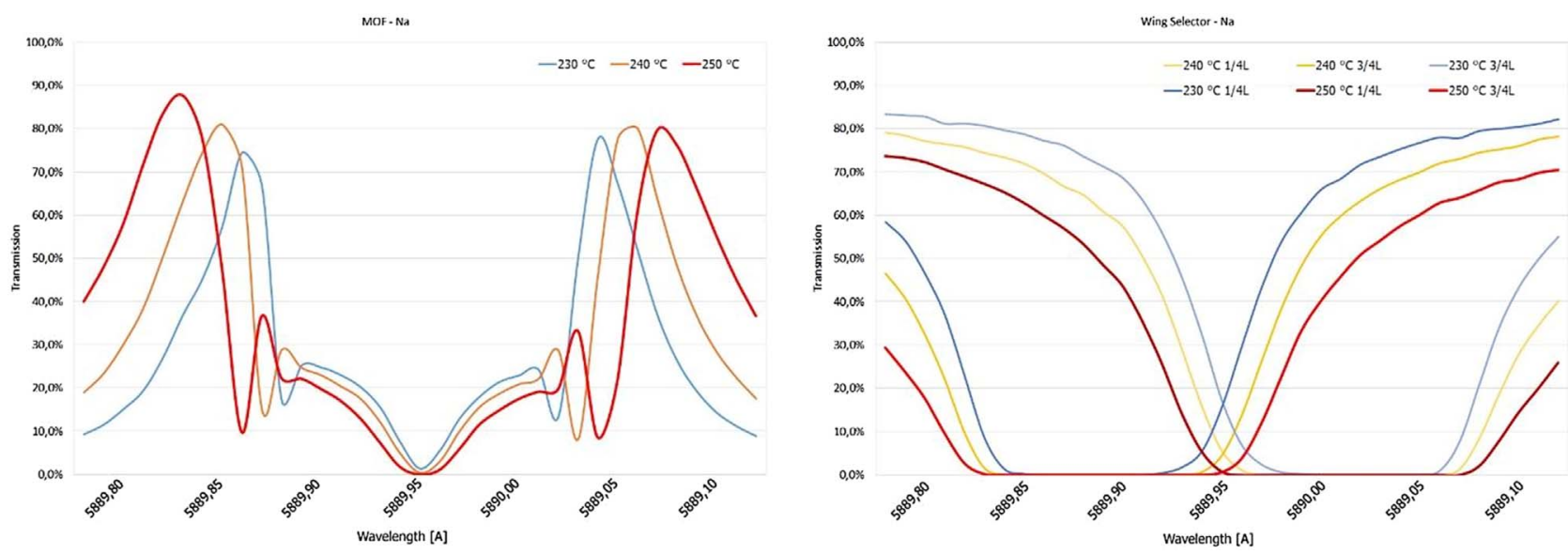

Fig. 5. Left: transmission profiles at three different temperatures of the MOF-Na filters (2000 G). Right: transmission profiles of the Wing Selector $(3700 \mathrm{G})$ taken at three different temperatures and in the two states of the LCVR (L/4 and 3L/4).


Fig. 6. Left: example of transmission profiles of the MOF-K filter taken during our several test with different temperatures a magnetic field fixed at $1400 \mathrm{G}$. This profiles are calibrated in wavelength only. The red curve, which is calibrated both in transmission and wavelength, matches the MOF we are using in its final configuration $\left(115^{\circ} \mathrm{C} / 1240 \mathrm{G}\right)$. Right: transmission profiles of the Wing Selector (2480 G) at three different temperatures and in the two states of the LCVR (L/4 and 3L/4).

market (Avalon Linear, M0 and M1) but specifically developed to handle the dual channel configuration and the weight of the SAMM Instrumentation $(70 \mathrm{~kg})$.

\subsection{The Merlino GUlz}

The Merlino GUI is mainly used to control the observatory and its systems, including the two SAMM channels. Besides to manual intervention on any device, this GUI allows to trigger pre-programmed macros such as the startup and shutdown ones that are configured to automatically initialize the instrument at the beginning of the operations and stop it at the end. Also emergencies, such as bad weather or remote control signal loss, are handled by this software that autonomously takes care of parking the telescope and closing the enclosure (Fig. 7).

\subsection{StarGo GUI}

The StraGo software manages the mount and the two optical tubes focusers. It is used to slew to the Sun and allows small pointing adjustments for an accurate centering of active regions. It is driven by ASCOM drivers and therefore could be interfaced to any compatible software just like the main SAMM control system (Fig. 8).

\subsection{The SAMM GUI}

The SAMM GUI has been developed by the INAF-OAR researchers using LabView. Since the main interface drives one single channel at time, two instances (one per channel) have to run in parallel. The interface presents controls for specific components of the polarimeters in well localized areas of the window, as well as buttons to trigger the coordinated actions on the entire channel, like for instance the launch of acquisition sequences (Fig. 9):

1. Action buttons - Apart from standby, that is the default status of the instrument, two alternative operating modes are available: Live View (used to prepare the instrument and interactively tune the operating parameters) and 


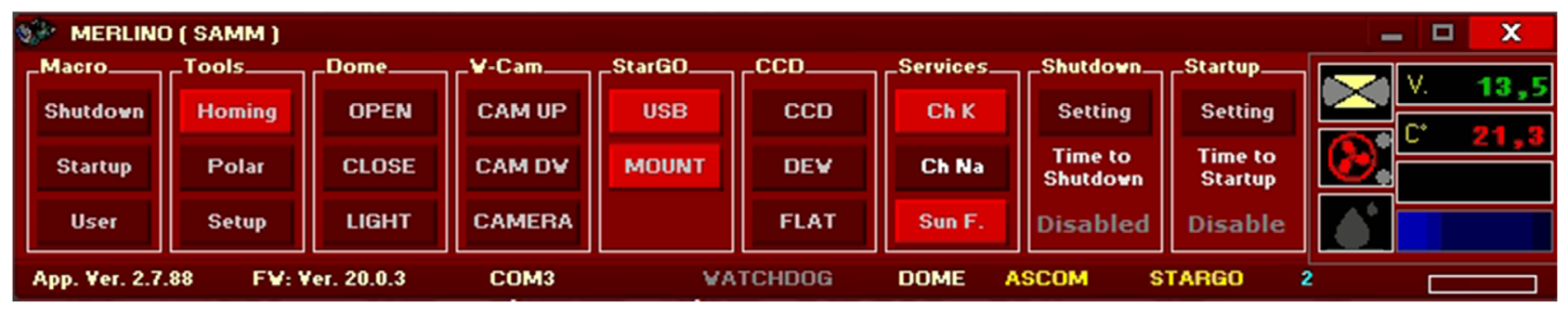

Fig. 7. The Merlino GUI.
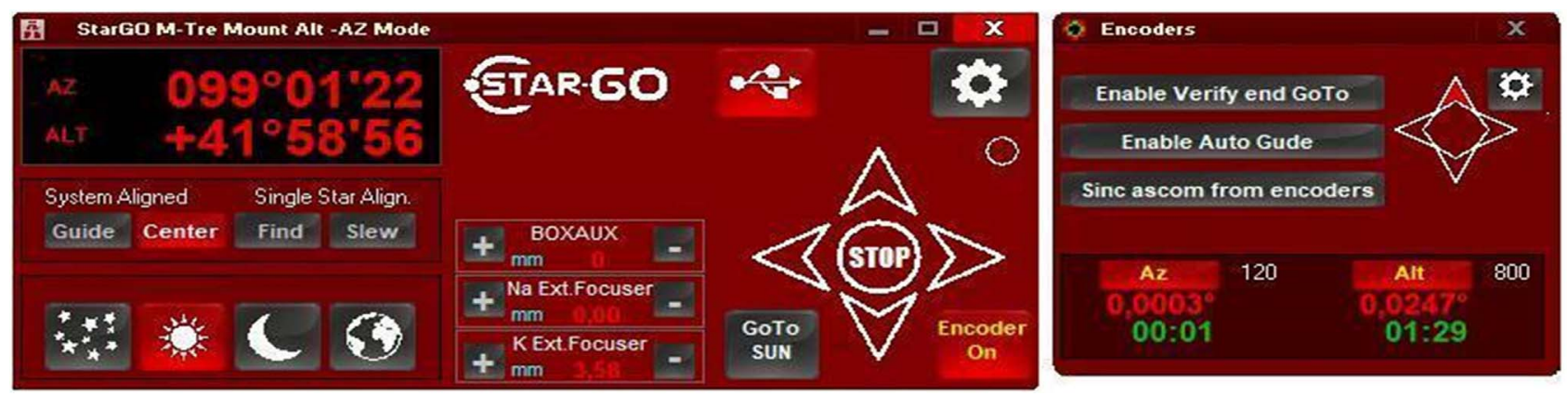

Fig. 8. The StarGo GUI.

Acquisition (to launch real acquisition with data saved on disk).

2. Image - The images coming from the camera appear in real time. Are displayed also the MIN, MAX, MEAN values calculated in the selection BOX defined by the two lines.

3. Temperature control - Here the observer checks the current temperatures and switches on/off the heaters of the MOF/WS cells. A graph with the target and the actual temperature is showed for any MOF. The two lines are superimposed when the target temperature is reached. The knob can be rotated to the "warm up" position when heating from ambient temperature to warm the cell to an intermediate temperature thus reducing the thermal stress on the cells.

4. LCVR - Settings of the retardance of the Liquid Crystal polarizers by varying the voltage applied to them (the two LCVRs have been calibrated on the bench to define the voltage values to be applied during the modulation exploited in a science acquisition session).

5. Motorized stages - With this interface it is possible to rotate the first linear polarizer placed in front of the MOF cell, and align it in parallel to the second linear polarizer to increase the throughput when the MOF is at ambient temperature, or to cross it when the MOF is working as a filter to cancel out the background. On the right side of this sub panel, the user can move the linear stage that holds the last lens of the optical train giving a fine adjustment of the focus.

6. Camera controls - Basic controls of the status of the camera. Here the user monitors the connection of the device and handles its cooling.

7. Acquisition - Is where the acquisition run is configured, the setup of the exposure time and of the number of images in a burst (images sequentially acquired for the same LCVR configuration) are selectable. On the bottom there is a counter that works as progress indicator. The fast mode is a special mode that reduces to the very minimum the system overheads such as image statistics and image quality calculations, devices feedback checks, to maximize images throughput and reduce the total acquisition time.

8. Configuration TABs - In each tab the user can set all the parameters of the hardware the GUI has to control, the addresses of the various devices, the destination path of the images and so on.

\section{Acquisition and data analysis}

The sequence of operations of a typical observing session is as follows:

(a) Turn on MOFs heating passing through the warm up stage.

(b) Select LCVR in Automatic mode to get them automatically change retardance during acquisition.

(c) Activate Live view mode to center the region of interest on the Sun, decide exposure time and fine tune focus.

(d) Start images acquisition.

Since short exposures of the order of 1-3 ms are required to minimize the smearing due to the air turbulence, a single set of images contains too few photons to achieve a reasonable SNR. As a consequence, the combination of several sets is required and, to achieve a high temporal resolution, these should be acquired in the shortest possible time. The current solution consists in the acquisition of 100 sets of images ( 1 set $=4$ images in the four LCVR states) for a total of 400 images acquired in about $80 \mathrm{~s}$. Being the instrument exposed to high thermal 


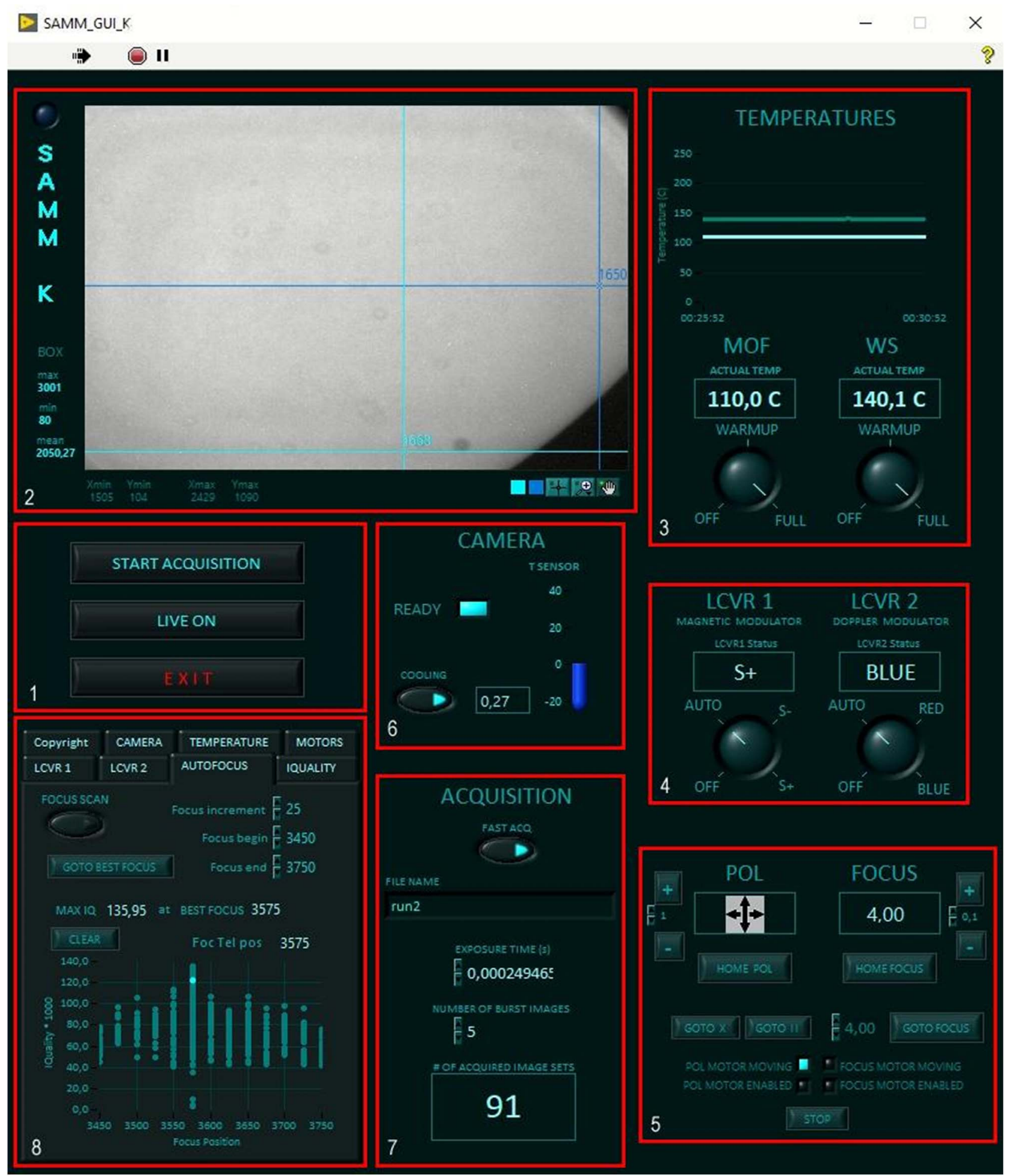

Fig. 9. The SAMM GUI.

gradients $\left(\Delta T \approx 10{ }^{\circ} \mathrm{C}\right)$ during the observation session that usually starts early in the morning, the control software automatically refines the focus before starting the acquisition of a new set of images.

To produce the expected observables, the acquired images are automatically processed by a data reduction pipeline that is triggered as soon as the acquisition run is complete. This pipeline at first takes care of standard data reduction operations on each single image (bias subtraction, flat fielding and fringes removal) and performs an image quality assessment by mean of a bi-dimensional DCT (discrete cosine transform) based algorithm. The selected best images from each LCVR status 

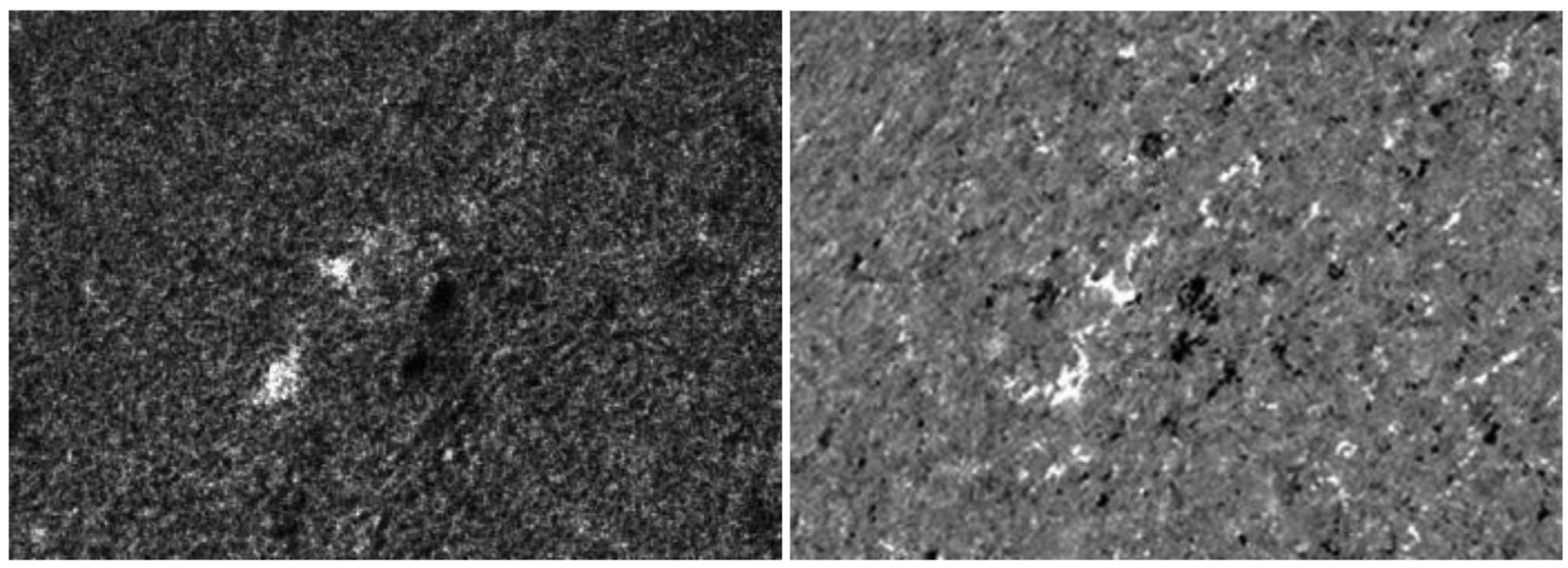

Fig. 10. Comparison between the K magnetogram of SAMM (left) and SDO (right) of the Active region 12,749. (Courtesy of NASA/SDO and the AIA, EVE, and HMI science teams). FOV: $5.5^{\prime} \times 3.9^{\prime}$.

are rotated, to compensate the alt-az tracking movement, co-aligned by means of image correlation and then composed to obtain a master image. Master images are then summed to get the total intensity and properly combined to produce a dopplergram and magnetogram. The data processing is performed in parallel to the acquisition procedure so we are able to release data in near real time with an overall cadence of $\leq 80 \mathrm{~s}$.

The pipeline software is constantly improving its performances in terms of data quality and processing speed with the aim to produce the final images before a new data run is available for processing.

\section{First images}

The commissioning of the SAMM telescope has been slowed down due to the Sun being at its minimum activity in the past year and the recent COVID-19 pandemic. Even if the magnetic structures were of the order of a few hundreds of Gauss and not strong enough to generate a solar spot on the photosphere, we were able to get the very first magnetograms and dopplergrams by developing a data reduction pipeline able to process all the acquired images by using the solar limb as a spatial reference for multiple images recentering.

Unluckily, due to a condensation problem of one of the two high speed cameras, we were able to carry on the commissioning of the Potassium channel only.

Even if the actual site of the SAMM (the roof of Avalon Instruments headquarters) is far from optimal in terms of seeing, the first images are a very encouraging and give an idea of the capability of the instrument when will be placed in an astronomical site. After several on sky tests we consider as the very first image the one obtained on the active region 12,749 taken on Oct 10th 2019 around 9:22 UTC (Fig. 10).

It comes from a 100 data sets taken with $2 \mathrm{~ms}$ exposure and processed with an early version of the data reduction pipeline. From the comparison with the SDO-HMI image of the same region taken almost at the same time, it is possible to detect magnetic features down to a few tens of Gauss while the brightest part of the feature has a magnetic field of the order of $500 \mathrm{G}$.

While the extended strong features are well seen, the small structures with little values of the magnetic field are less visible due to both the instrumental sensitivity (Table 1) and the smoothing effect of the observing site seeing. With a limit instrumental sensitivity of about $10 \mathrm{G}$ we expect to have a noise level of about $30 \mathrm{G}$ and the smoothed low magnetic fields can reach this level.

Other differences between the two images are also due to the formation heights of the signals that are not exactly the same. In fact, the HMI magnetograms are derived from 12 wavelength positions across the nominal $6173.3 \AA$ spectral line, 6 on the right and 6 on the left circularly polarized light, while the SAMM magnetograms are computed from 4 wavelength positions across the nominal $7698.96 \AA$ potassium line, 2 on the right and 2 on the left circularly polarized light (Fig. 11).

For the comparison of the Dopplergrams we can apply the same considerations as for the magnetograms, and we can assume a noise level of about $30 \mathrm{~m} / \mathrm{s}$. Because we are at solar limb, the dominating velocity fields along the Sun-Earth line-of-sight are the supergranulation, the solar rotation and a residual component of the solar oscillations. The brighter and darker structures are comparable and in the SDO/HMI Dopplergram have the values of $-2500 \mathrm{~m} / \mathrm{s}$ and of $+500 \mathrm{~m} / \mathrm{s}$ respectively. This means that SAMM are observing a velocity range of about $3000 \mathrm{~m} / \mathrm{s}$. The major difference between the images is the offset velocity present both in SAMM, which observes from the Earth, and HMI instrument, observing from SDO Spacecraft orbit.

The "second" light was obtained on the Active region 12,757 on Jan 27th 2020 around 8:14 UTC on a region that, according to SDO data, was showing a magnetic field ranging between $-1700 \mathrm{G}$ and $1300 \mathrm{G}$ (Fig. 12).

This time, the magnetic structures were more intense, with a clear geometry and generating small sunspot. We can almost apply the same considerations of Figure 10. Again our limiting factor is the seeing of the test site, but even if it was poor, we are still able to clearly detect features with less than $100 \mathrm{G}$. 



Fig. 11. Comparison between the K dopplergrams of SAMM (left) and SDO (right) in the same field of the Active region 12,749 (Courtesy of NASA/SDO and the AIA, EVE, and HMI science teams). Full frame: $10^{\prime} \times 8.3^{\prime}$.

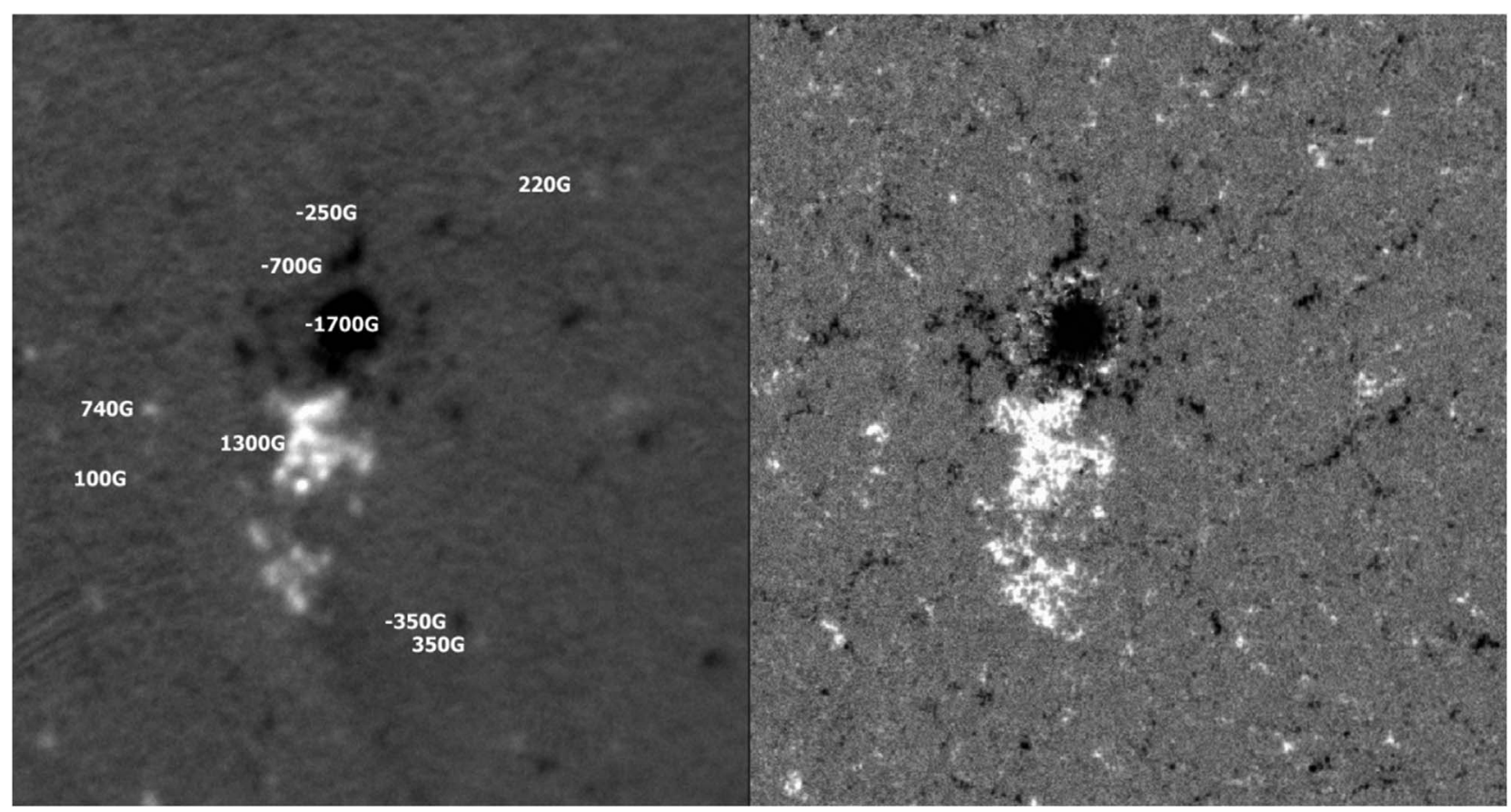

Fig. 12. Comparison between the K magnetogram of SAMM (left) and SDO (right) of the active region 12,757 (Courtesy of NASA/SDO and the AIA, EVE, and HMI science teams). FOV: $5.5^{\prime} \times 5.5^{\prime}$.

\section{Conclusions}

We have presented here the very first magnetograms and dopplergrams of the SAMM telescope. This project is an example of what a scientific collaboration between a research Institute and a private company can achieve. We could develop a new instrument based on a well-known technology already used in the past on a few occasions, and bring it to a pre-production level after a long engineering process. Since the second camera has just arrived after the C-19 lockdown, we expect to fully commissioning the binocular operation in a few months with the goal to have the instrument ready to the market by the end of the year. Once ready a SAMM station will offer the community a powerful and cost effective instrument to be replicated in a network and be able to deliver an unprecedented set of data in the space weather framework.

Acknowledgements. We'd like to express our special thanks to Prof. Robert von Fay-Siebenburgen from the University of Sheffield and Prof. Mihalis Mathioudakis of the Queen's 
University of Belfast for their early interest and support in this project. The editor thanks two anonymous reviewers for their assistance in evaluating this paper.

\section{References}

Agnelli G, Cacciani A, Fofi M. 1975. The magneto-optical filter, I. Preliminary observations in Na D lines. Sol Phys 44: 509. https://doi.org/10.1007/BF00153229.

Cacciani A, Fofi M. 1978. The magneto-optical filter, II. Velocity field measurements. Sol Phys 59: 179-189. https://doi.org/ 10.1007/BF00154941.

Cacciani A, Moretti PF, Rodgers WE. 1997. Measuring doppler and magnetic fields simultaneously. Sol Phys 174: 115-128. https://doi.org/10.1023/A:1004935524038.

Cacciani A, Moretti PF. 1994. Magneto-optical filter (MOF): concept and applications in astronomy. In: SPIE Proceedings 2198, Instrumentation in Astronomy VIII. https://doi.org/10.1117/12. 176751

Cacciani A, Jefferies SM, Finsterle W, Rapex P, Knox A, Giebink C, Di Martino V. 2003. A new instrument for sounding the solar atmosphere. In: Proceedings of SOHO 12/GONG+ 2002. Local and global helioseismology: The present and future, pp. 243-245. ISBN 92-9092-827-1. Bib. Code: 2003ESASP. 517..243C.

Hill F, Hammel H, Martinez-Pillet V, de Wijn A, Gosain S, et al. 2019. ngGONG - The next generation GONG - A new solar synoptic observational network. A White Paper for the Astro2020 Decadal Survey. Bib. Code: 1996AAS...188.5301L.

Kappenman J. 2010. Geomagnetic storms and their impacts on the U.S. power grid. Metatech Report. https://www.ferc.gov/industries/ electric/indus-act/reliability/cybersecurity/ferc_meta-r-319.pdf.

Leibacher JW, Harvey JW, Hill F, Hubbard R, Kennedy JR, Pintar JA, Bhatnagar A, Kennewell JA, Marquette W, Patron J, Saa O, Yasukawa E. 1996. The global oscillation network group (gong) project. Science 272(5266): 1284-1286. Bib. Code: 1996AAS... 188.5301L.

Lloyd's $360^{\circ}$ Risk. 2010. Space Weather. Its impact on Earth and implication for business. https://www.lloyds.com/news-and-riskinsight/risk-reports/library/natural-environment/space-weather.
Lloyd's Report. 2013. Solar storm risk to the North American electric grid. https://www.lloyds.com/news-and-risk-insight/riskreports/library/natural-environment/solar-storm.

Magrì M, Oliviero M, Severino G. 2008. Accurate intensity velocity phase difference in the potassium resonance line obtained with VAMOS. Sol Phys 247(1): 15-23. https://doi.org/10.1007/s11207007-9035-1.

Oliviero M, Moretti PF, Severino G, Straus T, Magrì M, Tripicchio A. 2002. Preliminary results on the solar photospheric dynamics observed with Vamos. Sol Phys 209(1): 21-35. https://doi.org/ 10.1023/A:1020904204842.

Oliviero M., Severino G., Straus T. 1998. The VAMOS data analysis pipeline. In: Structure and dynamics of the interior of the sun and sun-like stars SOHO, Boston, Massachusetts, 6/GONG 98, pp. 275-278. Bib. Code: 1998ESASP.418..275O.

Schou J, Scherrer PH, Bush RI, Wachter R, Couvidat S, et al. 2012. Design and ground calibration of the helioseismic and magnetic imager (HMI) Instrument on the solar dynamics observatory (SDO). Sol Phys 275: 229-259. https://doi.org/10.1007/s11207011-9842-2.

SCOR Papers. 2014. Solar storms and their impacts on power grids. Recommendations for (re)insurers. https://www.scor.com/en/file/ 15621/download?token=IBBlryDJ.

Severino G, Oliviero M, Landi Degl'Innocenti E. 2007. Simulation of Magneto-Optical Filter Transmission Profiles. In: Proceedings of the conference held 9-13 October, 2006 at the University of Coimbra in Coimbra, Portugal, 2007, Heinzel P, Dorotovič I, Rutten RJ (Eds.), Astronomical Society of the Pacific, San Francisco, Vol. 368, 617 p. Bib. Code: 2007ASPC..368..617S

Stangalini M, Piazzesi R, Speziali R, Dal Sasso L. 2018. SAMM: The solar activity MOF monitor. In: SPIE Proceedings 10700, Ground-based and Airborne Telescopes VII, 107001K. https://doi. org/10.1117/12.2313373.

Tomczyk S, Streander K, Card G, Elmore D, Hull H, Cacciani A. 1995. An instrument to observe low-degree solar oscillations. Sol Phys 159(1): 1-2. https://doi.org/10.1007/BF00733027.

Vogt E, Oliviero M, Severino G, Straus T. 1999. Calibration of VAMOS magnetic data. In: Proc. 9th European Meeting on Solar Physics, Magnetic Fields and Solar Processes, Florence, Italy, 12-18 September 1999, ESA SP-448, 405V. Bib Code: 1999ESASP.448..405V.

Cite this article as: Speziali R, Di Paola A, Centrone M, Oliviero M, Bonaccini Calia D, et al. 2021. The first light of the Solar Activity MOF Monitor Telescope (SAMM). J. Space Weather Space Clim. 11, 22. https://doi.org/10.1051/swsc/2020078. 